\title{
Using students' misconceptions of primary coloured lights to design a hands-on coloured light mixer
}

\section{Suchai Nopparatjamjomras, Ratchapak Chitaree}

Suchai Nopparatjamjomras, Ratchapak Chitaree, "Using students' misconceptions of primary coloured lights to design a hands-on coloured light mixer," Proc. SPIE 9666, 11th Education and Training in Optics and Photonics Conference, 966600 (5 June 2009); doi: 10.1117/12.2207964

SDIE Event: Eleventh International Topical Meeting on Education and Training in Optics and Photonics, 2009, St. Asaph, United Kingdom 


\title{
Using students' misconceptions of primary coloured lights to design a hands-on coloured light mixer
}

\author{
Suchai Nopparatjamjomras ${ }^{1}$, Ratchapak Chitaree $^{2}$ \\ ${ }^{1}$ Institute for Innovative Learning, Mahidol University, Rama VI Rd, Bangkok, 10400, \\ Thailand \\ ${ }^{2}$ Department of Physics, Mahidol University, Rama VI Rd, University, Bangkok, 10400, \\ Thailand \\ Author Contact: sumuetu37@yahoo.com
}

\begin{abstract}
A surface mount typed multi-coloured Light-Emitting Diode (LED) is used as a light source for the hands-on coloured light mixer. The LED consists of red, green and blue tiny sources but the mixer is designed to have four switches corresponding to red, green, blue and yellow light. These colours correspond to students' misconceptions of pimary coloured lights; they realize that the primary colours and the rules for lights mixing are the same as those of paints. To generate a yellow light, a microcontroller placed between four input switches and the LED operates both a red and green tiny sources. In addition, the microcontroller is employed to eliminate some combinations of coloured light mixing to simplify the experiment (basic mode) for non advanced students. If the mixer is used with more advanced students, a number of combinations will increase and students need more analytical skills to find out the primary coloured lights (the coloured lights that can not be produced by the mixing of any other coloured lights). Therefore, the mixer is able to use with more advanced and non advanced students depending on the program in the microcontroller and some modifications of the circuit. Furthermore, to introduce students an idea that other hues or shades can be generated by mixing of these three primary coloured lights of different intensities, a tuning circuit is integrated to vary an intensity of the green light source.
\end{abstract}

Keywords: coloured light mixer, hands-on, primary coloured lights, misconceptions

\section{INTRODUCTION}

A number of science educators presented that students come to a classroom with their "Prior knowledge". The knowledge is often opposite to the correct understanding of the nature. Then, it is teachers' responsibility to help enhance students changing alternative conception to scientific understanding. If the new knowledge fit with students' prior knowledge, it can be easily assimilated otherwise students need to shape those teacher give to them before they can accommodate 1. Therefore, an effective active learning teaching module should be designed based on students' prior knowledge so students can assimilate what the module give to them.

To survey students' prior knowledge, teachers can simply pose some questions to students or use a conceptual survey at the starting of a class. The examples of very well known conceptual survey are Conceptual Survey on Electricity and Magnetism (CSEM), Determining and Interpreting Resistive Electric Circuits Concepts Test (DIRECT), Force Motion Conceptual Evaluation (FMCE), Geosciences Concept Inventory $(\mathrm{GCl})$ and Heat and Temperature Conceptual Evaluation (HTCE). For more information, students' alternative conception can be found on the websites ${ }^{2}$. 
The alternative conceptions can be used as a guideline to design a learning track, what students need to observe or should be emphasized, to help students change their alternative conception to scientific understanding.

In this research, students' alternative conception of primary coloured lights, they realize that the

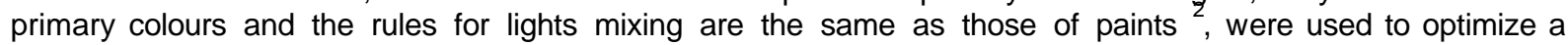
number of coloured lights and their mixing for a hands-on coloured light mixer. Furthermore, questions (what students need to Predict, Observe and Discuss) in an activity sheet were designed based on this alternative conception.

The mixing of lights can simply conducted by a light mixer. The mixer used 1) a microcontroller to limit a number of the available combinations of coloured light mixing and 2) a surface mount device (SMD) multi-colour LED (see figure 1) as the light sources. The LED can generate a uniform brightness light without any handwork because there is no epoxy drop lens on the top ${ }^{3}$. Moreover, it consists of three tiny red, green and blue light sources that are very close packed $(\sim 1 \mathrm{~mm}$ apart) so students perceive a mixing of these beams as a one beam (see figure 2).

The activity was designed based on PODS (Predict, Observe, Discuss and Synthesize) learning cycle ${ }^{4}$. Students were asked to predict a few questions on primary coloured lights and their mixing then they observed some prepared coloured lights mixing. These observations and discussions on the conflicts between students' prediction and observation with their peer helped them to correct their misconceptions. At the end of activity, a few questions were posed to students to confirm and enhance their scientific understanding.

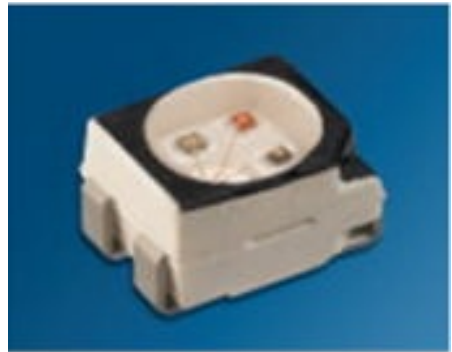

Figure 1: A SMD type multicolour LED
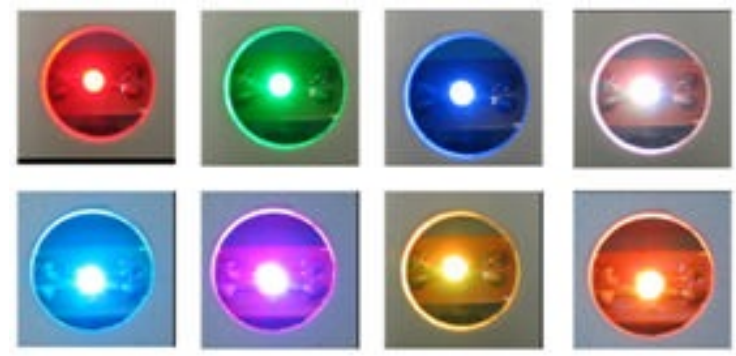

Figure 2: Coloured lights generated by the mixer (red, green, blue, white, cyan, magenta, yellow and orange).

\section{A HANDS-ON COLOURED LIGHT MIXER}

There are many research developed the instruments used to demonstrate primary coloured light mixing phenomena ${ }^{3,5-17}$. To design a simple hands-on coloured light mixer, a schematic diagram introduced by Planinšic (see figure 3 ) is used ${ }^{3}$. The circuitry of the mixer is very simple and consists of 1 ) three LEDs serve as red, green and blue light sources 2) three dimmers used to control the brightness of the three light sources, independently. In addition, three fix resistors ( $R 4, \mathrm{R} 5$ and R6) are used to limit the maximum current passing through a red, green and blue LED, respectively.

An LED is more appropriate to be used as a light source of a hands-on coloured light mixer than a bulb because it can be used with a battery and the price is cheaper than those of bulbs. This means that the LED based light mixer can be produced cheaply as a mass of teaching tools. However, LED still poses a problem. Most students hardly perceive a mixing of red, green and blue lights with the same intensities as 
white. The reason behind this problem are human resolving power ${ }^{18}$, a property of LED that is not a point source and can not generate uniform brightness ${ }^{3,19}$ and is easily disturbed by an ambient light ${ }^{20}$.

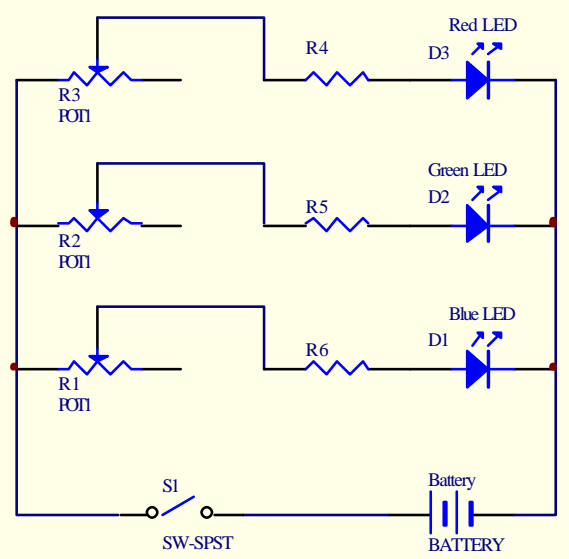

Figure 3: Schematic diagram of a simple coloured light mixer.

The easiest way to eliminate the problem of an ambient light is using the mixer in a dark room which is not available for most schools.

Gillies and Planinšic also suggested the way to change an LED to be a semi-point source that could generate a uniform brightness light. They used a hacksaw to saw the top part of the LED (function as a drop lens) off then polished the sawed surface with fine water sandpaper. Finally, an abrasive polishing paste was used to make the surface looks clearly transparent.

In addition, the problem of human resolving power can be eliminated by packing the light sources very close together or increasing an observing dstance between students and the mixer which is not suitable for a hands-on activity.

Fortunately, modern technology that always makes things easier is a success key of the hands-on coloured light mixer in this research. The surface-mount device LED so-called "SMD multi-coloured LED" is used as the light sources for the mixer. This multi-coloured LED has no lens on the top and consists of very closely packed three internal tiny of red, green, and blue light sources. Therefore, light produced by the LED is uniformly bright lights and viewed as one beam by the human eyes. Furthermore, the beams are bright enough to use in a normal-lit room.

Since the objective of this research is to encourage students to discover the primary colours of light but the simple mixer does not allow students to observe other coloured lights mixing except red, green and blue lights. Therefore, the mixer need to be modified to have more light sources then students can find out the primary colours of light; the coloured lights corresponding to students' definition of primary colours that they have already learned in the Art subject. This can be done by simply adding the extra light sources to the mixer but we need to face the fact that too much data make more difficult for students to analyze, especially the non advanced students. To optimize the problem, students' misconception about primary coloured lights can be used as a guide to provide the minimum light sources for the mixer.

As Bill Beaty posed on his website, many students realize that the primary colours and the rules for lights mixing are the same as those of paints, only four "students' possible primary colours of light (red, green, blue and yellow)" are used as the light sources of the modified mixer. All possible combinations of these four coloured lights of maximum intensities as well as their corresponding results are listed in Table 1 . The details 
indicate that only combinations 1 to 5 are enough for students to change their understanding about the primary colours of light and learn the conventional secondary colours of light. When students choose combination 1 (mixing red with green) they should realize immediately from observation that yellow is not a primary colour of light. Combinations 2 to 4 give magenta, cyan, and white light sequentially helping students learn the conventional secondary colours of light and the mixing of all primary coloured lights. Combination 5 should also convince students that the rule of coloured-light mixing is not the same as those of paint mixing; if it is, students would perceive the colour of the mixed lights as green. Therefore, the $1^{\text {st }}$ to $5^{\text {th }}$ combinations are provided for non-advanced students and the all combinations requiring more analytical skills to interpret is suitable for instruct more advanced students.

Table 1: All possible combinations of a red, green, blue and yellow lights mixing with the maximum intensities.

\begin{tabular}{|c|c|c|}
\hline Combination & Mixing of Coloured lights & \multicolumn{2}{|c|}{ Observed results } \\
\hline 1 & Red and Green & \\
2 & Red and Blue & \\
\hline 3 & Blue and Green & \\
\hline 4 & Red, Green, and Blue & \\
\hline 5 & Blue and Yellow & \\
\hline 6 & Red and Yellow & \\
\hline 7 & Green and Yellow & \\
\hline 8 & Red, Green, and Yellow & \\
\hline 9 & Red, Blue, and Yellow & \\
\hline 10 & Green, Blue, and Yellow & \\
\hline 11 & Red, Green, Blue, and Yellow & \\
\hline
\end{tabular}

To limit a number of available combinations of coloured light mixing, a microcontroller (AT89C2051) placed between four input switches and the multi-colour LED is employed to control the three very tiny LEDs, individually. Therefore, the available coloured light mixings are limited by the programme in the microcontroller. A flowchart of the microcontroller programme used in the mixer is presented in Figure 4. Executing the programme, which is run as a loop, needs inputs from a combination(s) of the four input switches. If the input matches, the pre-determined combinations coloured light can be observed. If there is no match, no light will be turned on. In addition, pressing the yellow light switch activated the microcontroller to turn both red and green LEDs on simultaneously at maximum intensities to give the yellow light. 


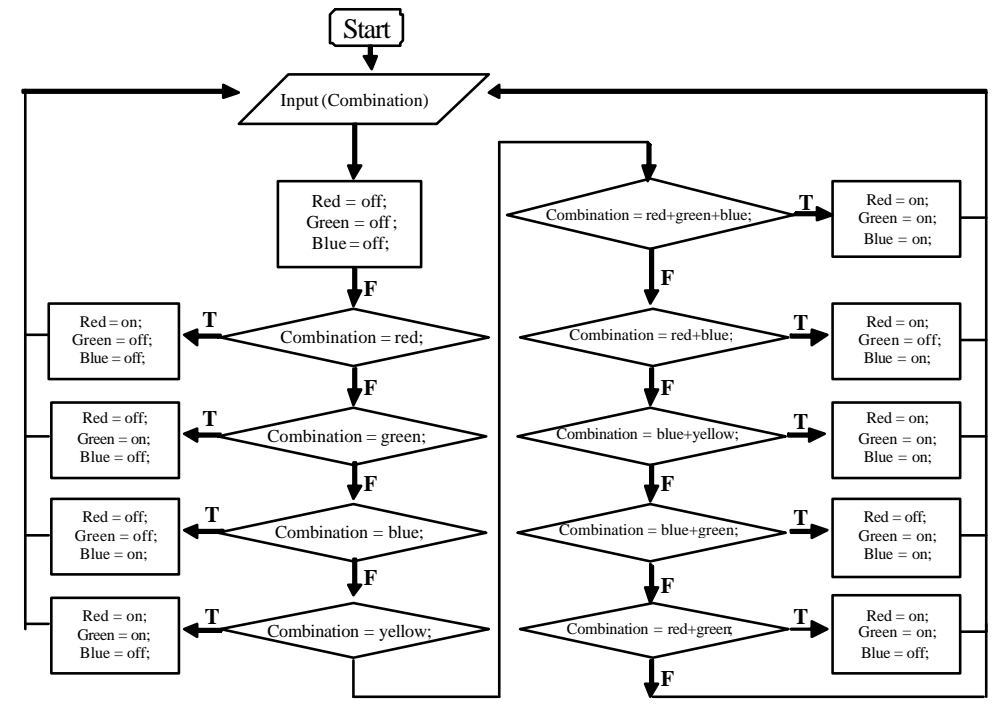

Figure 4: Flowchart of the microcontroller programme used in the hands-on coloured light mixer.

As students should be introduced to the idea that other hues or shades could be generated by mixing of these three primary coloured lights of different intensities, only one tuning circuit is added $\mathrm{b}$ make a green LED change its intensity by just turning he knob. This feature generates shades of colours; ranging from yellow to orange and pale blue to cyan. The schematic diagram and a complete set of the mixer are illustrated in Figure 5.

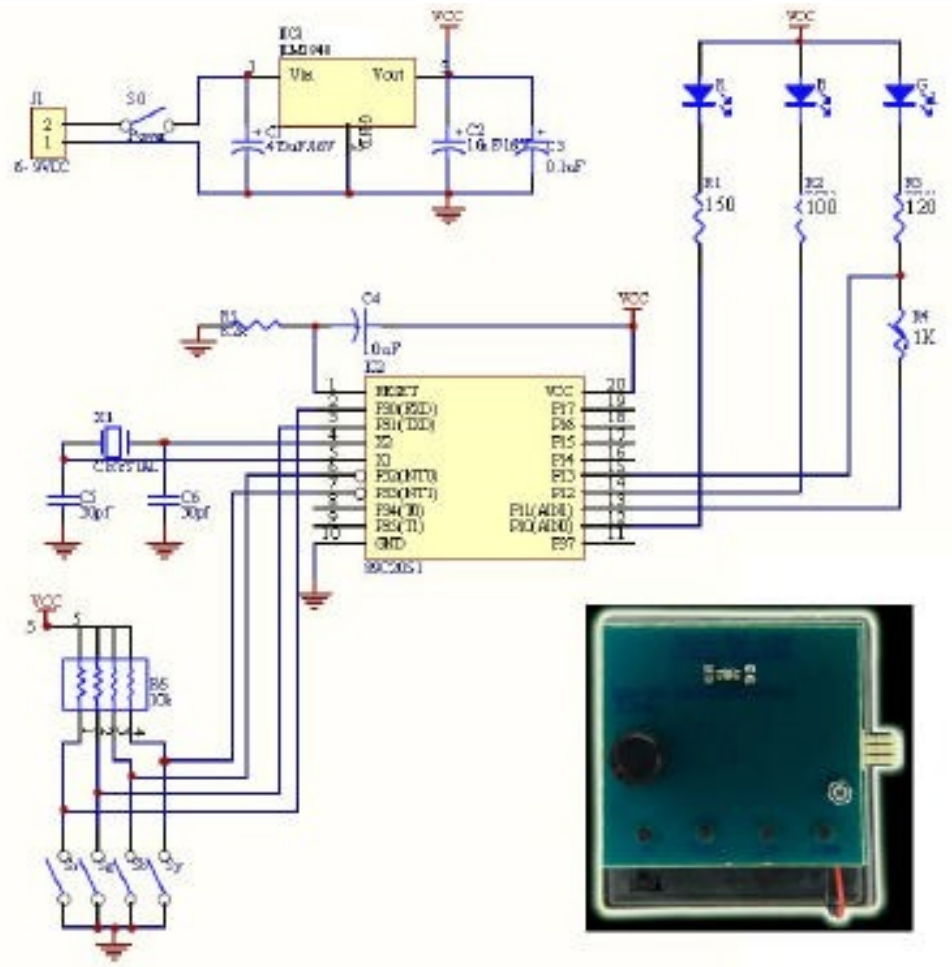

Figure 5: Schematic diagram and the third version of mixer. 


\section{ACTIVITY}

The 1 hour activity is designed based on the PODS learning cycle and the aims of the activity are encouraging students to discover, from their definition of primary colours, the primary colours of light and observing the secondary colours also the results from mixing of different intensity of lights.

The PODS learning cycle encourage students to learn from any contradiction between their predictions and observations that could be resolved during the discussion phase. Students then synthesize their newly learned ideas and conclusions into the more general framework of their physics knowledge. However, naïve students should be given some background information in the form of simple introductory activities to increase their confidence to make a prediction rather than just making a random or uninformed guesses. This is because the background information is a backup for students to use logical reasoning to make their predictions.

The activity is started with the classroom discussion to reach an agreement on the definition of primary colours that is "Combination of two primary colours cannot produce a third primary colour when all of them are added together, the white light will be produced". Students then are asked to predict what the colours are the primary colours of light.

Before students observe some prepared coloured light mixing, they have to predict and write down their predictions in the prediction column of the activity sheet (see Table 2). Students then do the experiment themselves on the five combinations of coloured light mixing with the same intensities as mentioned in the second section. The combinations consist of 1) red mixed with blue 2) red mixed with green 3) green mixed with blue 4) blue mixed with yellow 5) red and green mixed with blue. Moreover, students have to observe the other combination which is red and green light with the intensity of green is less than those of red.

After that students discuss with their peers on these questions 1) why is yellow not a primary colour of light? 2) What are the primary colours of light? In addition, students have to consider the correctness of this sentence "if an object reflects red, green and blue lights with the same intensities to an eye, colour of the object will be perceived as white".

In the synthesis phase, students have to think about the relation between the primary colours of light and the colour sensitivity of the colour receptors in the human eye and also the colour of the white screen perceived by the red blindness person.

Table 2: Table of prediction and Observation.

\begin{tabular}{|l|l|l|}
\hline \multicolumn{1}{|c|}{ Questions } & $\begin{array}{l}\text { Your group } \\
\text { prediction }\end{array}$ & Observation \\
\hline $\begin{array}{l}\text { 1. The colour of light produced by the mixing of red light } \\
\text { and blue lights with the maximum intensity is...... }\end{array}$ & & \\
\hline $\begin{array}{l}\text { 2. The colour of light produced by the mixing of red light } \\
\text { and green lights with the maximum intensity is...... }\end{array}$ & & \\
\hline $\begin{array}{l}\text { 3. The colour of light produced by the mixing of green } \\
\text { light and blue lights with the maximum intensity is...... }\end{array}$ & & \\
\hline $\begin{array}{l}\text { 4. The colour of light produced by the mixing of blue } \\
\text { light and yellow lights with the maximum intensity } \\
\text { is...... }\end{array}$ & & \\
\hline $\begin{array}{l}\text { 5. The colour of light produced by the mixing of red } \\
\text { light, green light and blue lights with the maximum } \\
\text { intensity is...... }\end{array}$ & & \\
\hline $\begin{array}{l}\text { 6. The colour of light produced by the mixing of red light } \\
\text { and green with the green intensity is less than red } \\
\text { intensity is...... }\end{array}$ & & \\
\hline
\end{tabular}




\section{EFFICACY OF THE HANDS-ON COLOURED LIGHT MEXER}

To evaluate an efficacy of the teaching module, the module was introduced and conducted by the researcher to four classes of 151, Year-11 female students. Students' response to three questions of primary coloured light and their mixing collected before and two weeks after a class was determined in term of an average normalized gain. This gain is the ratio of the actual average gain to the maximum possible average gain ${ }^{21}$ as

$$
<>=\frac{\left(\%<S_{f}>-\%<S_{i}>\right)}{100-\%<S_{i}>}
$$

where $<S_{f}>$ and $<S_{i}>$ are the final (post) and initial (pre) class averages

The questions are 1) what colour(s) are the primary colour(s) of light? 2) Is this sentence correct, "the mixing of blue and yellow light on a white paper can not be perceived as green"? and 3) Can orange light be generated by the mixing of red and green light?

Figure 6 presents students' response to the first question. This reveals that only $30 \%$ of students have a correct prior knowledge of the primary colours of light. While the other group of students (30\%) believes that the primary colours of light are the same as the primary colours of paints. Some students (11\%) have the idea that the primary colours of light should be the same as colours of a rainbow. These results also support the reason behind the development of the mixer that a large number of students are confused the three primary colours of light (red, green, blue) with those of paints (red, yellow, blue).

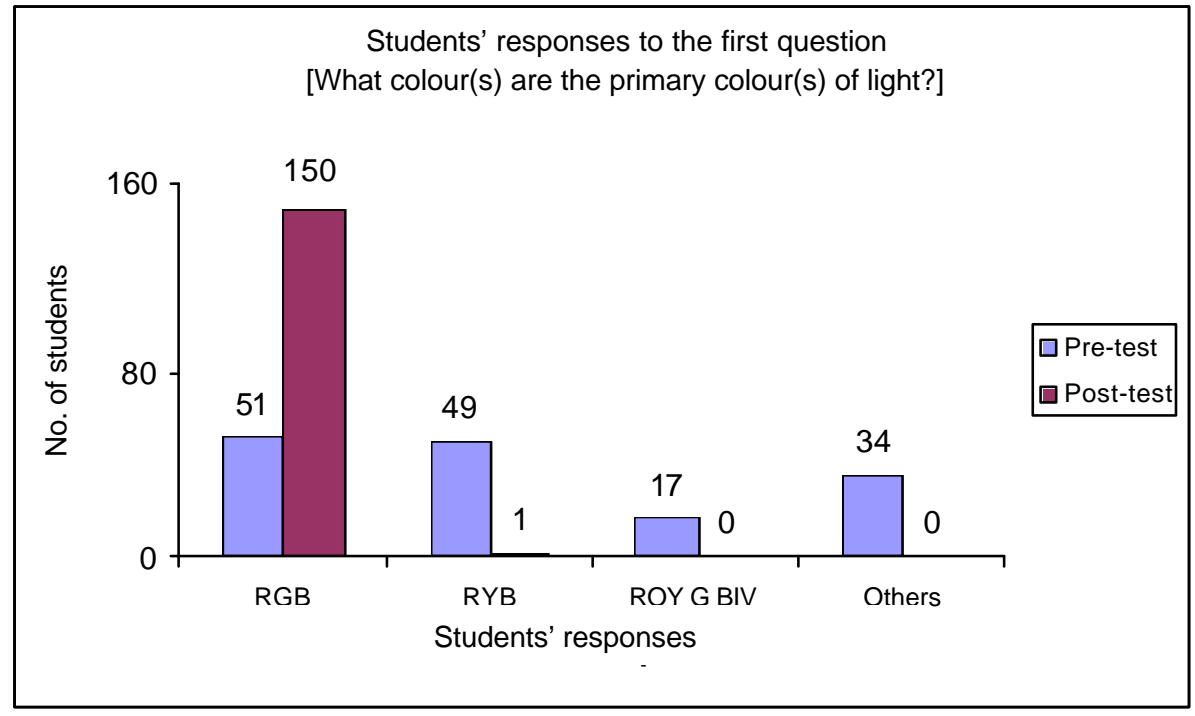

Figure 6: Students' responses to the first question.

Furthermore, students' responses to the second and third questions as shown in figure 7 indicate that there is only $15 \%$ of students before the instruction know the mixing of blue and yellow light can not generate green light and some students (27\%) realize that orange light can be generated by the mixing of red and green light. 


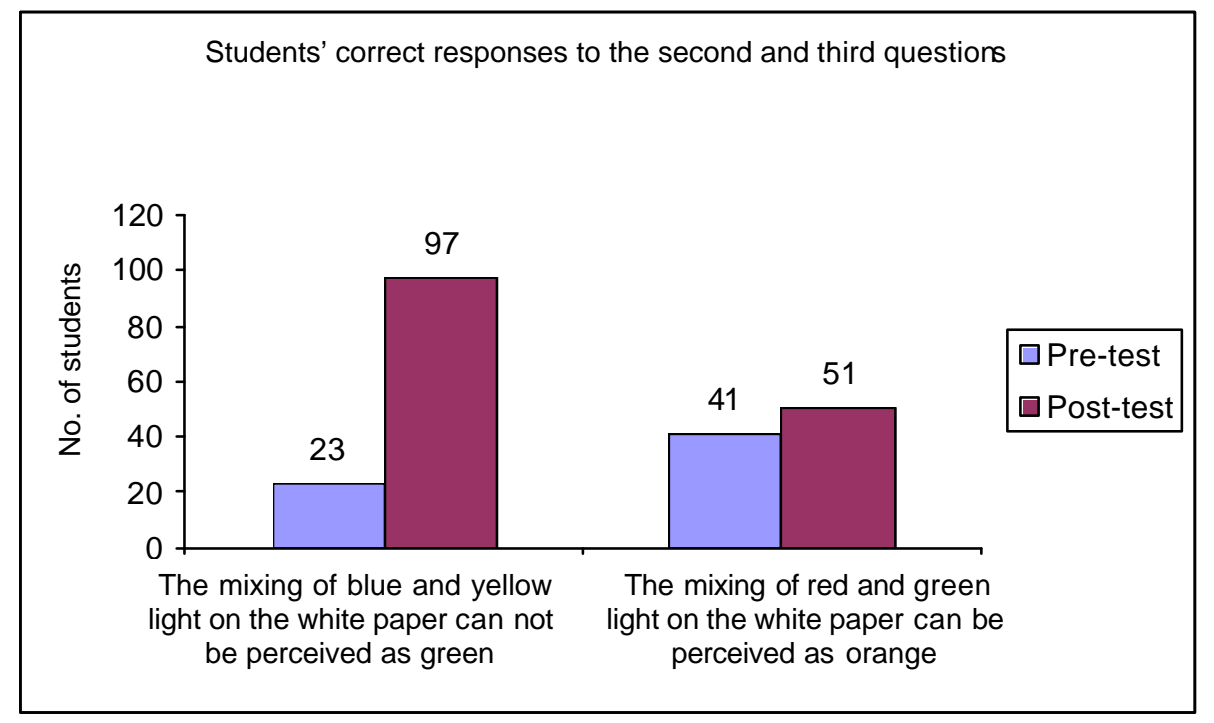

Figure 7: Students' correct response to the second and third questions.

After completion of the instruction, the number of students who have a correct understanding of primary coloured lights and their mixing is significantly increased. The average normalized gain corresponding to the $1^{\text {st }}-3^{\text {rd }}$ questions are $0.99,0.58$, and 0.09 , respectively.

\section{CONCLUSION}

The hands-on coloured light mixer developed in this research could eliminate the problems found on the previous research as 1) a requiring of a dimly lit or dark room that was difficult to provide for general schools 2) some modifications of light sources are needed. This could be achieved by employing two modern technologies: a SMD multi-coloured LED set and a microcontroller. The LED has no epoxy drop lens on the top and consists of three tiny red, green and blue light sources that are very closely packed. Therefore, the output beams are uniform brightness and viewed as one beam. In addition, a number of the available combinations of coloured light mixing are limited by the programme in the microcontroller. If the mixer is used with more advanced students, the programme and the circuit need some modifications. Moreover, the mixer was designed to use as a hands-on instrument with an ative learning based teaching module to encourage students to investigate the primary colours of light, the coloured lights corresponding with their definition of primary colours, and their mixing. This could help students learn science actively rather than passively.

\section{ACKNOWLEDGMENTS}

A gradate fellowship from the Institute for Promoting Science and Technology for S.N. is gratefully acknowledged. The authors would like to thank the Faculty of Graduate Studies, Mahidol University for financial support. 


\section{Reference:}

[1] Peter W. Hewson, Michael E. Beeth and N. Richard Thorley, "Teaching for Conceptual Change". In B.J. Fraser and K.G. Tobin(eds.), International Handbook of Science Education. (Kluwer Academic Publishers, Netherlands, 1998).

[2] Bill Beaty, "Ttite," (1998).

[3] Gorazd Planinšic, "Color Mixer for Every Student," The Physics Teacher 42 (3), 138-142 (2004).

[4] Z. B. Lakhadar, I. B. Culaba, V. Lakshminarayanan, J. T. Maquiling, A. Mazzolini, and D. R. Sokoloff, Active Learning in Optics and Photonics Training Manual. (UNESCO, Paris, 2006).

[5] Richard A. Bartels, "Using a video projector for color-mixing demonstrations," The Physics Teacher 20 (4), 247-248 (1982).

[6] Richard A. Bartels, "A hallway display of additive color mixing," The Physics Teacher 24 (9), 564-565 (1986).

[7] William A. Butler, A. Douglas Davis, and Jr Charles E. Miller, "Color mixing for a large audience," The Physics Teacher 17 (1), 43-43 (1979).

[8] Chris Chiaverina, "The Ping-Pong Ball Color Mixer Revisited," The Physics Teacher 45 (2), 120-120 (2007).

[9] John Cockman, "A Bright Color Mixer," The Physics Teacher 40 (9), 553-553 (2002).

[10] Jr Thomas B. Greenslade, "The von Nardroff Color Mixing Apparatus," The Physics Teacher 43 (9), 602-602 (2005).

[11] John J. Heilemann, "A Lantern Slide Color Mixer," The American Physics Teacher 3 (4), 184-184 (1935).

[12] John J. Heilemann, "An Indicating Lantern Slide Color Mixer," The American Physics Teacher 4 (4), 211-211 (1936).

[13] Marinus G. Luttikhuizen, "Color mixing using a single projector," The Physics Teacher 26 (5), 296-297 (1988).

[14] J. A. Maroto, C. M. Penalver Valverde, and J. E. Bueno-Gonzalez Tejero, "Description of additive colour mixing exhibits by using PC-designed Maxwell discs," Physics Education 41 (5), 448-452 (2006).

[15] A. Alan Middleton and Samuel Sampere, "Color mixing via polarization," The Physics Teacher 39 (2), 123-124 (2001).

[16] Leonard Parsons, "As easy as R, G, B," The Physics Teacher 36 (6), 347-348 (1998).

[17] Larry Russell, "Color mixing with lasers," The Physics Teacher 39 (8), 475-475 (2001).

[18] Daniel A. Dale and Brenae L. Bailey, "Physics in the Art Museum," The Physics Teacher 41 (2), $82-83$ (2003).

[19] George T. Gillies, "Altered lightemitting diode point source emitter," American Journal of Physics 48 (5), 418-418 (1980).

[20] D. H. Brainard, "Ttite," (Elsevier Science Ltd., 2001), Vol. 2008.

[21] Richard R. Hake, "Interactive-engagement versus traditional methods: A six-thousand-student survey of mechanics test data for introductory physics courses," American Journal of Physics 66 (1), 64-74 (1998). 